\title{
MIXED FINITE ELEMENT METHODS FOR ELLIPTIC PROBLEMS*
}

\author{
DOUGLAS N. ARNOLD $\dagger$
}

\begin{abstract}
This paper treats the basic ideas of mixed finite element methods at an introductory level. Although the viewpoint presented is that of a mathematician, the paper is aimed at practitioners and the mathematical prerequisites are kept to a minimum. A classification of variational principles and of the corresponding weak formulations and Galerkin methods - displacement, equilibrium, and mixed —is given and illustrated through four significant examples. The advantages and disadvantages of mixed methods are discussed. The concepts of convergence, approximability, and stability and their interrelations are developed, and a résumé is given of the stability theory which governs the performance of mixed methods. The paper concludes with a survey of techniques that have been developed for the construction of stable mixed methods and numerous examples of such methods.
\end{abstract}

Key words. mixed method, finite element, variational principle

1. Introduction. The term mixed method was first used in the 1960's to describe finite element methods in which both stress and displacement fields are approximated as primary variables. We begin with the most classical example, the system of linear elasticity.

The equations of linear elasticity consist of the constitutive equation

$$
A \mathcal{S}=\mathcal{E}(\mathbf{u}) \text { in } \Omega
$$

and the equilibrium equation

$$
\operatorname{div} \mathcal{S}=\mathbf{f} \text { in } \Omega
$$

Here $\Omega$ denotes the region in three dimensional space, $\mathbb{R}^{3}$, occupied by the elastic body, $\mathbf{u}: \Omega \rightarrow \mathbb{R}^{3}$ denotes the displacement field, $\mathcal{E}(\mathbf{u})$ denotes the corresponding infinitesimal strain tensor, (i.e., the symmetric part of the gradient of $\left.\mathbf{u}, \epsilon_{i j}(\mathbf{u})=\left(u_{i, j}+u_{j, i}\right) / 2\right)$ ), $\mathbf{f}$ denotes the imposed volume load, and $\mathcal{S}: \Omega \rightarrow \mathbb{R}_{s}^{3 \times 3}$ (the space of symmetric $3 \times 3$ tensors) denotes the stress field. The divergence of $\mathcal{S}, \operatorname{div} \mathcal{S}$, is applied to each row of $\mathcal{S}$, so that $(\boldsymbol{d i v} \mathcal{S})_{i}=\sum_{j} s_{i j, j}$. The material properties are determined by the compliance tensor $A$ which is a positive definite symmetric operator from $\mathbb{R}_{s}^{3 \times 3}$ to itself, ${ }^{1}$ possibly depending on the point $\boldsymbol{x} \in \Omega$. The constitutive equations can equally well be written as

$$
\mathcal{S}=C \mathcal{E}(\mathbf{u}) \text { in } \Omega
$$

*This work was supported by NSF grant DMS-89-02433.

$\dagger$ Department of Mathematics, The Pennsylvania State University, University Park, Pennsylvania 16827 .

${ }^{1}$ This means that the action of $A$ can be written as $(A \mathcal{S})_{i j}=\sum_{k l} a_{i j k l} s_{k l}$ with the components $a_{i j k l}$ satisfying the usual major symmetries $a_{i j k l}=a_{k l i j}$, minor symmetries $a_{i j k l}=a_{j i k l}$, and positivity condition $\sum_{i j k l} a_{i j k l} s_{i j} s_{k l} \geq \gamma \sum_{i j} s_{i j}^{2}$, for all $\mathcal{S}$, where $\gamma>0$. 
where the elasticity tensor $C: \mathbb{R}_{s}^{3 \times 3} \rightarrow \mathbb{R}_{s}^{3 \times 3}$ is the inverse of $A$. This example also serves to illustrate our font conventions: vector quantities are notated in boldface, second order tensors are in script, and fourth order tensors are sans serif.

To determine a unique solution, we supplement the elasticity equations by the boundary conditions

$$
\left.\mathbf{u}\right|_{\Gamma_{d}}=\mathbf{g}_{d} \quad \text { and }\left.\quad \mathcal{S} \mathfrak{n}\right|_{\Gamma_{t}}=\mathbf{g}_{t}
$$

where $\Gamma_{d}$ and $\Gamma_{t}$ are complementary parts of $\partial \Omega$ and $\mathbf{g}_{d}$ and $\mathbf{g}_{t}$ give the displacements and tractions prescribed on $\Gamma_{d}$ and $\Gamma_{t}$ respectively.

Mixed methods for the elasticity problem are mostly based on the following mixed variational principle which is a form of the Hellinger-Reissner principle:

The solution $(\mathcal{S}, \mathbf{u})$ of the elasticity problem can be characterized as the unique critical point of the functional

$$
L(\mathcal{T}, \boldsymbol{v})=\int_{\Omega}\left(\frac{1}{2} A \mathcal{T}: \mathcal{T}+\operatorname{div} \mathcal{T} \cdot \boldsymbol{v}-\mathbf{f} \cdot \boldsymbol{v}\right)-\int_{\Gamma_{d}} \mathbf{g}_{d} \cdot(\mathcal{T} \mathfrak{n})
$$

over the space of all symmetric tensorfields $\mathcal{T}$ satisfying the traction boundary condition $\left.\mathcal{T} \mathbf{n}\right|_{\Gamma_{t}}=\mathbf{g}_{t}$, and all vectorfields $\boldsymbol{v}$.

Indeed, if we set the first variation of $L$ with respect to $\mathcal{T}$ equal to zero, we get the equation

$$
\int_{\Omega}(A \mathcal{S}: \mathcal{T}+\operatorname{div} \mathcal{T} \cdot \mathbf{u})=\int_{\Gamma_{d}} \mathbf{g}_{d} \cdot(\mathcal{T} \mathbf{n})
$$

for all $\mathcal{T}$ for which $\mathcal{T} \mathfrak{n}$ vanishes on $\Gamma_{t}$. Integrating by parts, we obtain the constitutive equation and displacement boundary condition. Taking the variation of $L$ with respect to $\boldsymbol{v}$ leads immediately to the equilibrium equation. Note that in the form of the HellingerReissner principle presented, the traction boundary condition is essential -it is imposed a priori on the space where the stress tensor is sought — while the displacement boundary condition arises naturally from the variational principle.

To make the variational principle precise, we must state over what space of functions $\mathcal{T}$ and $\boldsymbol{v}$ are to vary. The appropriate choice for $\mathcal{T}$ is the subspace of $\mathcal{H}(\mathbf{d i v})$ (symmetric tensorfields which are square integrable and have square integrable divergence) of fields satisfying the traction boundary condition, and for $\boldsymbol{v}$ the space $\mathbf{L}^{2}$ of all square integrable vectorfields. The reader who is uncomfortable with these function spaces need not be concerned: suffice it to say that they are chosen in a fairly natural way so that the integrals involved in the definition of $L$ make sense.

A key point, which is characteristic of mixed variational principles, is that the pair $(\mathcal{S}, \mathbf{u})$ is not an extreme point of the Hellinger-Reissner functional. It is a saddle point. In fact

$$
L(\mathcal{S}, \boldsymbol{v}) \leq L(\mathcal{S}, \mathbf{u}) \leq L(\mathcal{T}, \mathbf{u})
$$


for all $\mathcal{T} \in \mathcal{H}(\mathbf{d i v})$ satisfying $\left.\mathcal{T} \mathfrak{n}\right|_{\Gamma_{t}}=\mathbf{g}_{t}$ and all $\mathbf{u} \in \mathbf{L}^{2}$. It follows from this saddle point condition that

$$
\sup _{\boldsymbol{v} \in \mathbf{L}^{2}} \inf _{\substack{\left.\mathcal{T} \in \mathcal{H}(\mathbf{d i v}) \\ \mathcal{T} \mathfrak{n}\right|_{\Gamma_{t}}=\mathbf{g}_{t}}} L(\mathcal{T}, \boldsymbol{v})=L(\mathcal{S}, \mathbf{u}) \quad \text { and } \quad \inf _{\substack{\left.\mathcal{T} \in \mathcal{H}(\mathbf{d i v}) \\ \mathcal{T} \mathfrak{n}\right|_{\Gamma_{t}}=\mathbf{g}_{t}}} \sup _{\boldsymbol{v} \in \mathbf{L}^{2}} L(\mathcal{T}, \boldsymbol{v})=L(\mathcal{S}, \mathbf{u})
$$

Now, because $\mathbf{u}$ satisfies the constitutive equation, $\mathcal{E}(\mathbf{u})$ is square integrable. It follows (from Korn's inequality) that the gradient of $\mathbf{u}$ is square integrable, i.e., $\mathbf{u} \in \mathbf{H}^{1}$. Let us set, for any $\boldsymbol{v} \in \mathbf{L}^{2}$,

$$
E(\boldsymbol{v})=-\inf _{\substack{\left.\mathcal{T} \in \mathcal{H}(\mathbf{d i v}) \\ \mathcal{T} \mathbf{n}\right|_{\Gamma_{t}}=\mathbf{g}_{t}}} L(\mathcal{T}, \boldsymbol{v})
$$

Then $E(\mathbf{u})=-L(\mathcal{S}, \mathbf{u})$ and we have from (1) that

$$
-E(\mathbf{u})=\sup _{\boldsymbol{v} \in \mathbf{L}^{2}}-E(\boldsymbol{v})
$$

and, a fortiori,

$$
-E(\mathbf{u})=\sup _{\boldsymbol{v} \in \mathbf{H}^{1}}-E(\boldsymbol{v})
$$

or

$$
E(\mathbf{u})=\inf _{\boldsymbol{v} \in \mathbf{H}^{1}} E(\boldsymbol{v}),
$$

i.e., the displacement field $\mathbf{u}$ is characterized as the minimizer of the functional $E$ over $\mathbf{H}^{1}$. We shall show in a moment that for any $\boldsymbol{v} \in \mathbf{H}^{1}$

$$
E(\boldsymbol{v})= \begin{cases}\int\left(\frac{1}{2} C \mathcal{E}(\boldsymbol{v}): \mathcal{E}(\boldsymbol{v})+\mathbf{f} \cdot \boldsymbol{v}\right)-\int_{\Gamma_{t}} \mathbf{g}_{t} \cdot \boldsymbol{v}, & \text { if }\left.\boldsymbol{v}\right|_{\Gamma_{d}}=\mathbf{g}_{d} \\ \infty, & \text { otherwise }\end{cases}
$$

This permits us to interpret (2) as the following variational priniciple, which is nothing but the usual prinicipal of minimal potential energy energy.

The displacement field $\mathbf{u}$ solving the elasticity problem minimizes the functional

$$
\int_{\Omega}\left(\frac{1}{2} C \mathcal{E}(\boldsymbol{v}): \mathcal{E}(\boldsymbol{v})+\mathbf{f} \cdot \boldsymbol{v}\right)-\int_{\Gamma_{t}} \mathbf{g}_{t} \cdot \boldsymbol{v}
$$

over the space of all vectorfields satsifying the displacement boundary conditions.

Thus, starting from the Hellinger-Reissner mixed principle, we have derived the standard displacement variational principle. Note that for the latter the displacement boundary condition is essential, and the traction condition natural. 
To verify (3) we integrate by parts to get

$$
L(\mathcal{T}, \boldsymbol{v})=\int_{\Omega}\left(\frac{1}{2} A \mathcal{T}: \mathcal{T}-\mathcal{T}: \mathcal{E}(\boldsymbol{v})-\mathfrak{f} \cdot \boldsymbol{v}\right)+\int_{\Gamma_{t}} \mathbf{g}_{t} \cdot \boldsymbol{v}+\int_{\Gamma_{d}}\left(\boldsymbol{v}-\mathbf{g}_{d}\right) \cdot(\mathcal{T} \mathfrak{n})
$$

Now if $\boldsymbol{v}-\mathbf{g}_{d}$ doesn't vanish on $\Gamma_{d}$, then we may take $\mathcal{T}$ such that $\mathcal{T} \mathfrak{n}$ is an arbitrarily large negative multiple of this quantity on $\Gamma_{d}$, and we can arrange as well that $\mathcal{T}$ decay quickly away from $\partial \Omega$ so that its $L^{2}$ norm is arbitrarily small. It follows that $L(\mathcal{T}, \boldsymbol{v})$ can be made negative with arbitrarily large magnitude by appropriate choice of $\mathcal{T}$. Thus, if $\left.\boldsymbol{v}\right|_{\Gamma_{d}} \neq \mathbf{g}_{d}$, then $E(\boldsymbol{v})=\infty$. On the other hand, if $\left.\boldsymbol{v}\right|_{\Gamma_{d}}=\mathbf{g}_{d}$, then

$$
L(\mathcal{T}, \boldsymbol{v})=\int_{\Omega}\left(\frac{1}{2} A \mathcal{T}: \mathcal{T}-\mathcal{T}: \mathcal{E}(\boldsymbol{v})-\mathfrak{f} \cdot \boldsymbol{v}\right)+\int_{\Gamma_{t}} \mathbf{g}_{t} \cdot \boldsymbol{v}
$$

This quantity is clearly minimal when $A \mathcal{T}=\mathcal{E}(\boldsymbol{v})$, i.e, when $\mathcal{T}=C \mathcal{E}(\boldsymbol{v})$, and in this case

$$
L(\mathcal{T}, \boldsymbol{v})=\int_{\Omega}\left(-\frac{1}{2} C \mathcal{E}(\boldsymbol{v}): \mathcal{E}(\boldsymbol{v})-\mathbf{f} \cdot \boldsymbol{v}\right)+\int_{\Gamma_{t}} \mathbf{g}_{t} \cdot \boldsymbol{v}
$$

as claimed.

We have seen how the stress field can be eliminated from the mixed variational principle, leaving a variational characterization of the displacement. In a similar (simpler) way we can eliminate the displacement and obtain the following variational characterization of the stress: of all tensorfields which satisfy the equilibrium equation and the traction boundary conditions, $\mathcal{S}$ minimizes the complementary energy functional

$$
E_{c}(\mathcal{T})=\int_{\Omega} \frac{1}{2} A \mathcal{T}: \mathcal{T}-\int_{\Gamma_{d}} \mathbf{g}_{d} \cdot(\mathcal{T} \mathbf{n})
$$

These three basic variational principles for linear elasticity are summarized in Table 1. For each of these variational principles, the critical point is determined by the vanishing of the first variation, which leads to a weakly formulated boundary value problem. The weak formulations corresponding to our three variational principles are given in Table 2 .

Each of the three variational principles may be discretized by seeking a critical point of the relevant functional over a finite dimensional subspace (presumably of finite element type) of the admissable trial functions. Equivalently, in the weak formulations we can substitute the function spaces $\left(\mathbf{H}^{1}, \mathcal{H}(\mathbf{d i v})\right.$, and $\left.\mathbf{L}^{2}\right)$ with finite dimensional subspaces. The resulting discretization methods are termed Galerkin methods. For the primal principle the resulting Galerkin methods are termed displacement methods. For the dual principle such methods are commonly referred to as equilibrium methods. For the mixed variational principle we obtain mixed methods. In all three cases, the determination of the discrete solution ultimately reduces to the solution of a finite system of algebraic equations. 
Primal variational principle. Among all kinematically admissable vectorfields the displacement field is the unique critical point of the energy. This critical point is a minimum. I.e., $\mathbf{u} \in \mathbf{H}^{1},\left.\mathbf{u}\right|_{\Gamma_{d}}=\mathbf{g}_{d}$, and

$$
E(\mathbf{u})=\inf _{\substack{\left.\boldsymbol{v} \in \mathbf{H}^{1} \\ \boldsymbol{v}\right|_{\Gamma_{d}}=\mathbf{g}_{d}}} E(\boldsymbol{v})
$$

Dual variational principle. Among all statically admissable tensorfields the stress field is the unique critical point of the complementary energy. This critical point is a minimum. I.e., $\mathcal{S} \in \mathcal{H}(\mathbf{d i v})$, $\operatorname{div} \mathcal{S}=\mathbf{f},\left.\mathcal{S} \mathbf{n}\right|_{\Gamma_{t}}=\mathbf{g}_{t}$, and

$$
E_{c}(\mathbf{u})=\inf _{\substack{\mathcal{T} \in \mathcal{H}(\mathbf{d i v}) \\ \operatorname{div} \mathcal{T}=\left.\mathbf{f} \\ \mathcal{T} \mathbf{n}\right|_{\Gamma_{t}}=\mathbf{g}_{t}}} E_{c}(\mathcal{T})
$$

Mixed variational principle. Among all tensorfields assuming the prescribed tractions on $\Gamma_{t}$ and all vectorfields, the stress and displacement fields give the unique critical point of the Hellinger-Reissner functional $L$. This critical point is a saddle point. I.e., $\mathcal{S} \in \mathcal{H}(\boldsymbol{d i v})$, $\left.\mathcal{S} \mathbf{n}\right|_{\Gamma_{t}}=\mathbf{g}_{t}, \mathbf{u} \in \mathbf{L}^{2}$, and

$$
\sup _{\boldsymbol{v} \in \mathbf{L}^{2}} L(\mathcal{S}, \boldsymbol{v})=L(\mathcal{S}, \mathbf{u})=\inf _{\substack{\left.\mathcal{T} \in \mathcal{H}(\mathbf{d i v}) \\ \mathcal{T} \mathbf{n}\right|_{\Gamma_{t}}=\mathbf{g}_{t}}} L(\mathcal{T}, \mathbf{u})
$$

TABLE 1. Basic variational principles for linear elasticity.

2. Other examples. Most of the elliptic problems arising from mathematical physics and engineering admit analogous variational formulations. We list some of these here. For simplicity we ignore the boundary conditions.

The scalar second order elliptic problem

$$
\mathcal{A} \mathbf{s}=\operatorname{grad} u, \quad \operatorname{div} \mathbf{s}=f
$$

which models, e.g., a stationary thermal distribution with temperature field $u$ and flux field $\mathbf{s}$, is entirely analogous to the linear elasticity problem.

The Kirchhoff-Love plate model may be written

$$
A \mathcal{M}=-\mathcal{G} \mathcal{R} \mathcal{A D} \operatorname{grad} w, \quad-\operatorname{div} \operatorname{div} \mathcal{M}=f
$$


Primal problem. Find $\boldsymbol{u} \in \mathbf{H}^{1}$ such that $\left.\mathbf{u}\right|_{\Gamma_{d}}=\mathbf{g}_{d}$, and

$$
\int_{\Omega} \mathcal{C E}(\mathbf{u}): \mathcal{E}(\boldsymbol{v})=-\int_{\Omega} \mathbf{f} \cdot \boldsymbol{v}+\int_{\Gamma_{t}} \mathbf{g}_{t} \boldsymbol{v}
$$

for all $\boldsymbol{v} \in \mathbf{H}^{1}$ such that $\left.\boldsymbol{v}\right|_{\Gamma_{d}}=0$.

Dual problem. Find $\mathcal{S} \in \mathcal{H}(\mathbf{d i v})$ such that $\operatorname{div} \mathcal{S}=\mathbf{f},\left.\mathcal{S} \mathfrak{n}\right|_{\Gamma_{t}}=\mathbf{g}_{t}$ and

$$
\int_{\Omega} A \mathcal{S}: \mathcal{T}=\int_{\Gamma_{d}} \mathbf{g}_{d} \cdot(\mathcal{T} \mathbf{n})
$$

for all $\mathcal{T} \in \mathcal{H}(\mathbf{d i v})$ such that $\operatorname{div} \mathcal{T}=0$ and $\left.\mathcal{T} \mathfrak{n}\right|_{\Gamma_{t}}=0$.

Mixed problem. Find $\mathcal{S} \in \mathcal{H}(\mathbf{d i v})$ satisfying $\left.\mathcal{S} \mathfrak{n}\right|_{\Gamma_{t}}=\mathbf{g}_{t}$ and $\mathbf{u} \in \mathbf{L}^{2}$ such that

$$
\int_{\Omega}(A \mathcal{S}: \mathcal{T}+\operatorname{div} \mathcal{T}: \mathbf{u}+\operatorname{div} \mathcal{S}: v)=\int_{\Omega} \mathfrak{f} \cdot \boldsymbol{v}+\int_{\Gamma_{d}} \mathbf{g}_{d} \cdot(\mathcal{T} \mathfrak{n})
$$

for all $\mathcal{T} \in \mathcal{H}(\mathbf{d i v})$ satisfying $\left.\mathcal{T} \mathbf{n}\right|_{\Gamma_{t}}=0$ and all $\boldsymbol{v} \in \mathbf{L}^{2}$.

TABLE 2. Weak formulations associated with the three variational principles.

(Here $(\mathcal{G R} \mathcal{A D} \operatorname{grad} w)_{i j}=w_{, i j}$ is the matrix of second partial derivatives and $\operatorname{div} \operatorname{div} \mathcal{M}=$ $\sum_{i j} m_{i j, i j}$.) The mixed variational principle characterizes the moment tensor $\mathcal{M}$ and the transverse displacement $w$ as a saddle point of the functional

$$
\int\left(\frac{1}{2} A \mathcal{M}: \mathcal{M}+w \operatorname{div} \operatorname{div} \mathcal{M}+f w\right)
$$

whereas the primal principle asserts that $w$ minimizes the energy functional

$$
\int\left(\frac{1}{2} C(\mathcal{G R} \mathcal{A D} \operatorname{grad} w): \mathcal{G R} \mathcal{A D} \operatorname{grad} w-f w\right)
$$

where $C=A^{-1}$.

In Stokes flow, the velocity $\mathfrak{u}$ and pressure $p$ satisfy

$$
\operatorname{div} C \mathcal{E}(\mathbf{u})+\operatorname{grad} p=f, \quad \operatorname{div} \mathbf{u}=0
$$


Together they are a saddle point of the the functional

$$
\int\left(\frac{1}{2} \mathcal{C E}(\mathbf{u}): \mathcal{E}(\mathbf{u})+p \operatorname{div} \mathbf{u}+f u\right) .
$$

For Stokes problems the primal variational principle, which characterizes the pressure independently of the velocity, is rarely used. This is because it involves the inversion of the differential operator div $C \mathcal{E}(\cdot)$, which is rarely practical. On the other hand, equilibrium methods, based on the dual principle that $\boldsymbol{u}$ minimize

$$
\int\left(\frac{1}{2} C \mathcal{E}(\mathbf{u}): \mathcal{E}(\mathbf{u})+f u\right)
$$

over divergence-free fields, are occasionally used.

The mixed weak formulations for all four examples are listed in Table 3. For simplicity we continue to ignore boundary conditions, and also do not insist on the precise function spaces involved. Notice the characteristic form shared by all the examples.

3. Advantages and disadvantages of mixed methods. A number of reasons have been put forth to prefer mixed methods over displacement or equilibrium methods in some situations. First of all, equilibrium methods are rarely used in practical computation due to the difficulty of creating finite element spaces incorporating the necessary constraints (the conditions of static admissability and, in particular, the equilibrium condition in the case of elasticity). Thus the practical choice is usually between the primal-based displacement methods and the mixed methods. For some problems, such as the Stokes problem, primalbased methods are impractical. For such problems the mixed methods are the simplest and most direct alternative and are widely used.

For the other examples, however, the most basic methods are primal-based. A commonly stated reason to prefer mixed methods in these cases is that the dual variable (stress for elasticity, flux for thermal problems, moments for plate bending) is often the variable of most interest. For primal-based methods this variable is not a fundamental unknown and must be obtained a posteriori by differentiation, which entails a loss of accuracy. For the mixed methods, however, the dual variable is computed as a fundamental unknown. Of course, this argument is only heuristic. Its correctness depends on the available mixed finite element spaces and primal finite element spaces, the accuracy they offer, and the computational work they require to solve the corresponding discrete problems.

Another common motivation for the use of mixed methods is the avoidance of $C^{1}$ elements for plate bending and other fourth order problems. This is because the mixed functional for plate bending involves no more than two derivatives in any term and hence, after a suitable integration by parts, may be evaluated on finite element spaces with merely continuous elements. The primal variational functional, however, requires the use of $C^{1}$ elements (or non-conforming elements). 
Elasticity: Find $\mathcal{S}$ and $\mathbf{u}$ such that

$$
\int_{\Omega}(A \mathcal{S}: \mathcal{T}+\operatorname{div} \mathcal{T} \cdot \mathbf{u}+\operatorname{div} \mathcal{S} \cdot \boldsymbol{v})=\int_{\Omega} \mathfrak{f} \cdot \boldsymbol{v}
$$

for all $\mathcal{T}$ and $\boldsymbol{v}$.

Scalar second order: Find $\mathbf{s}$ and $u$ such that

$$
\int_{\Omega}(\mathcal{A} \mathbf{s}: \mathbf{t}+\operatorname{div} \mathbf{t} \cdot u+\operatorname{div} \mathbf{s} \cdot v)=\int_{\Omega} f \cdot v
$$

for all $\mathbf{t}$ and $v$.

Plate: Find $\mathcal{M}$ and $w$ such that

$$
\int_{\Omega}(A \mathcal{M}: \mathcal{N}+\operatorname{div} \operatorname{div}: \mathcal{N} w+\operatorname{div} \operatorname{div}: \mathcal{M} v)=\int_{\Omega} f v
$$

for all $\mathcal{N}$ and $v$.

Stokes: Find $\mathbf{u}$ and $p$ such that

$$
\int_{\Omega}[C \mathcal{E}(\mathbf{u}): \mathcal{E}(\boldsymbol{v})+\operatorname{div} \boldsymbol{v} p+\operatorname{div} \mathbf{u} q]=\int_{\Omega} \mathbf{f} \cdot \boldsymbol{v}
$$

for all $v$ and $q$.

TABLE 3. Mixed weak formulations for various problems.

Another advantage of mixed variational principles is their robustness in the presence of certain limiting and extreme situations. For example, in the case of linear elasticity, the compliance tensor $A$ becomes singular in the limit of incompressibility. Consequently its inverse, the elasticity tensor $C$, blows up: it is very large for nearly incompressible materials and infinite for incompressible ones. (E.g., in the istropic case

$$
A \mathcal{T}=\frac{1+\nu}{E}\left(\mathcal{T}-\frac{\nu}{1+\nu} \operatorname{tr}(\mathcal{T}) \mathcal{I}\right), \quad C \mathcal{T}=\frac{E}{1+\nu}\left(\mathcal{T}+\frac{\nu}{1-2 \nu} \operatorname{tr}(\mathcal{T}) \mathcal{I}\right)
$$

with $\mathcal{I}$ denoting the identity matrix and tr the matrix trace operator. As the Poisson ratio $\nu \uparrow 1 / 2, A$ tends nicely to the limiting value

$$
A \mathcal{T}=\frac{3}{2 E}\left(\mathcal{T}-\frac{1}{3} \operatorname{tr}(\mathcal{T}) \mathcal{I}\right)
$$


but $C$ blows up.) A analogous situation holds for the Reissner-Mindlin plate model where the robustness is with respect to the plate thickness. Robustness properties of mixed methods have also been reported in other situations, as well. That is, mixed methods have been observed to perform significantly better than closely related displacement methods in particular applications that involve some extreme or limiting behavior. For example, Ewing, Wheeler, and others have reported superior computations of pressure (which satisfies a scalar second order elliptic problem arising from Darcy's law) via mixed methods when simulating the miscible displacement of oil from a porous media [12], [13]. Marini and Savini [20] have reported improved results in semiconductor device modelling through the use of mixed methods. In each case, the mixed methods seem to be exhibiting greater robustness with respect to the roughness of the coefficients of the equations. (In both problems there is a sharply defined front across which the coefficients change rapidly.)

In addition to the situations in which mixed methods are used explicitly, there are a number of methods which have been proposed in the literature which, while resembling displacement methods, can be shown to be equivalent to mixed methods. Such methods are called generalized displacement methods since they lead to discrete systems involving only degrees of freedom associated to the primal variable. However the discrete system differs from what would be obtained by straightforward discretization of the primal variational principle. The best known examples are the reduced and selective integration methods in which all or some of the terms of the primal energy functional are intentionally integrated with low accuracy. This apparently paradoxical procedure of reducing the integration accuracy in order to increase the solution accuracy was poorly understood and quite controversial when first introduced. In almost every case where it is successful, however, it can be shown that such a method is equivalent to a rather natural mixed method with exact, or at least accurate, integration [19]. In a number of cases the theory of mixed methods can be applied to provide a complete understanding and justification of reduced integration procedures. (Cf. [1] where the theory of mixed methods is used to give a complete analysis of reduced integration and standard displacement methods for the Timoshenko beam problem and [18] where some reduced integration methods for the Stokes problem and Reissner-Mindlin plate are analyzed as mixed methods.) A similar situation holds for other generalized displacement methods, such as ones involving harmonic averaging of rough coefficients [6] and interpolation [8] in the computation of the stiffness matrix. In addition a number of non-conforming displacement methods can be viewed, and best analyzed, as mixed methods [2]. In our view, this constitutes one of the most important roles of the theory of mixed methods: it provides tools to design and analyze high performance generalized displacement methods.

There are also obvious disadvantages to mixed methods in comparison with displacement methods. Because both the primal and dual variable are approximated simultaneously, the discrete system will typically involve many more degrees of freedom than a displacement method which uses a similar space to approximate the primal variable (but 
does not directly approximate the dual variable). Morever the fact that the primal variational principle is an extremal principle is reflected as positivity of the discrete system. Thus displacement methods for all the problems we have considered lead to positive definite algebraic systems. Since the mixed variational principal is a saddle point principal rather than an extremal principal the discrete system will be indefinite, possessing both positive and negative eigenvalues. Consequently a number of solution methods, both direct methods such as Cholesky decomposition and iterative methods like conjugate gradients, can not be applied directly.

Both these objection can often be overcome in practice by implementing mixed methods as generalized displacement methods. A simple case is when the finite element space for the dual variable does not incorporate any interelement continuity, i.e., all the degrees of freedom associated with the dual variable are internal to the elements. In this case the dual variable can be eliminated at negligible cost (by static condensation). The resulting system involves only the primal degrees of freedom and is positive definite. In fact many reduced integration methods arise in this way. More generally, when all the degrees of freedom of the dual variable are either interior to elements or lie on element edges (in two dimensions) or faces (in three dimensions) - but not at vertices - there is a quite general procedure to eliminate them at little cost [2], [15]. In contrast to the completely discontinuous case, this procedure adds additional degrees of freedom for the primal variable. The generalized displacement methods which arise typically use nonconforming elements.

A third possible objection to mixed methods is that they are subject to possible instabilities which do not arise for standard displacement methods. Thus the finite element spaces used to discretize extremal variational principles may be selected considering only their approximation properties and convenience of implementation. However for mixed variational principles, when spaces are selected on this basis alone they will almost always give poor results. For good convergence, the spaces must also satisfy some rather subtle stability conditions. Consequently the theory of mixed methods is more involved (and more interesting) than for displacement methods, and the design of effective mixed methods requires more expertise than for displacement methods. The stability properties of mixed methods, which form the heart of their mathematical theory, will be the subject of the remainder of this paper.

4. Approximability, stability, and convergence of Galerkin methods. All the weak formulations we have considered - primal, dual, and mixed - can be written in the form

$$
\text { Find } u \in V \text { such that } B(u, v)=F(v) \text { for all } v \in V \text {, }
$$

where $V$ is some function space, $B: V \times V \rightarrow \mathbb{R}$ is a bilinear form, and $F: V \rightarrow \mathbb{R}$ is a linear form.* Indeed any linear problem arising from a variational principle (i.e.,

\footnotetext{
*If the problem involves inhomogeneous (i.e., nonzero) essential boundary conditions, then $u$ here
} 
any problem in which the solution is characterized as a critical point of some quadratic functional) has this form (although the weak form is more general - it applies to problems that don't have a variational principle). To solve such a problem by a Galerkin method, we choose a finite-dimensional subspace $V_{h}$ of $V$ (typically a space spanned by a convenient set of finite element shape functions), and determine the approximate solution $u_{h}$ by the the same weak formulation, except that both the trial space where the approximate solution is sought and the space of test functions over which $v$ varies are replaced by $V_{h}$ :

Find $u_{h} \in V_{h}$ such that $B(u, v)=F(v)$ for all $v \in V_{h}$.

If the weak formulation arises from a variational principle, as in our examples, this is equivalent to discretizing the variational principle by seeking a critical point in the subspace $V_{h}$.

We shall be concerned with three properties of such Galerkin discretizations. Convergence measures the the smallness of the error $u-u_{h}$ between the exact solution and discrete solution. Good convergence properties are the fundamental goal of any numerical method. Approximability measures the error in the best approximation of $u$ by elements of $V_{h}$, i.e., the smallest possible error between the exact solution $u$ and any element of the discretization space $V_{h}$. Note that approximability depends on the choice of the space $V_{h}$ and the exact solution $u$, but not on the particular problem under consideration. The convergence achieved by a method is clearly limited by the approximability of the subspace, but good approximability does not guarantee good convergence. The missing ingredient turns out to be stability, which refers to the continuity of the mapping from the data $F$ to the discrete solution $u_{h}$.

To quantify these notions it is necessary to introduce norms to measure differences between functions. Let $\|v\|$ denote a norm on functions $v \in V$ (this simply means that $\|v\|$ is positive for any nonzero $v$, and that triangle inequality and the homogeneity condition $\|c v\|=|c|\|v\|$ hold). We will always assume that the norm is chosen so that the bilinear form $B$ is bounded, i.e., that there is a constant $K$ such that

$$
B(v, w) \leq K\|v\|\|w\|
$$

for all $v$ and $w$ in $V$. It is usually straightforward in practice to choose a norm so that (5) holds with $K$ not unreasonably large. For example if

$$
B(v, w)=\int a \operatorname{grad} v \cdot \operatorname{grad} w+\mathbf{b} \cdot \operatorname{grad} v w+c v w
$$

represents not the solution but rather the difference between the solution and some other, arbitrarily chosen but fixed, function satisfying the essential boundary conditions. To avoid this technical complication, which is not relevant here, we shall henceforth assume that any essential boundary conditions are homogeneous. 
the natural choice is

$$
\|v\|=\sqrt{\int|\operatorname{grad} v|^{2}+|v|^{2}},
$$

which is the Sobolev $H^{1}$ norm, and then $K$ would depend in a simple way on bounds for the coefficients $a, b$, and $c$. For the form

$$
B((\mathbf{s}, u),(\mathbf{t}, v))=\int \mathcal{A} \mathbf{s}: \mathbf{t}+\operatorname{div} \mathbf{t} \cdot u+\operatorname{div} \mathbf{s} \cdot v
$$

the natural choice of norm is

$$
\|(\mathbf{s}, u)\|=\sqrt{\int|\operatorname{div} \mathbf{s}|^{2}+|\mathbf{s}|^{2}+|u|^{2}} .
$$

This is the $H\left(\right.$ div) norm on $\mathbf{s}$ and the $L^{2}$ norm on $u$. The norms which arise naturally in this way are usually of practical significance. E.g., for displacement methods for elasticity, the natural norm is the energy norm. Of course we may be interested in the convergence of our method in other norms than the natural one (for example, we may be interested in the maximum of the stress rather than its root mean square). Convergence analysis in other norms than the natural one is possible, but involves further complications, and it is usually necessary to understand the convergence in the "natural norm" as a first step. In this paper we shall only consider convergence in the natural norm for the problem.

Having introduced a norm on $V$ it is clear how to measure convergence and approximability, namely by the quantities

$$
\left\|u-u_{h}\right\| \quad \text { and } \quad \inf _{v \in V_{h}}\|u-v\|
$$

respectively. To quantify the notion of stability we must also have a norm on the space of functionals on $V$. For this purpose we use the dual norm defined by

$$
\|F\|_{*}=\sup _{0 \neq v \in V} \frac{\|F v\|}{\|v\|}
$$

for $F: V \rightarrow \mathbb{R}$. Then the stability constant for the Galerkin method is given by

$$
C_{h}=\sup _{F: V \rightarrow \mathbb{R}} \frac{\left\|u_{h}\right\|}{\|F\|_{*}}
$$

That is, for any data $F$ we consider the solution $u_{h}$ to the discrete problem, and measure the size of $u_{h}$ compared to the size of $F$. The largest value this ratio achieves, for any possible data $F$, is the stability constant. If we think of the discrete problem as a matrix equation, then the stability constant is just the norm of the inverse matrix. With this 
notation, we can state the fundamental relation between convergence, approximation, and stability:

$$
\left\|u-u_{h}\right\| \leq K C_{h} \inf _{v \in V_{h}}\|u-v\|
$$

Since the constant $K$ will generally not be large if the norm is chosen reasonably, this relation says that if the stability constant $C_{h}$ is not large, then the error in the Galerkin solution will not be much larger than the error in the best approximation. To clarify this further, consider a sequence of subspaces $V_{h}$ parametrized by a positive number $h$ tending to zero (which could, for example, represent the mesh size, as in the standard finite element method, or the the inverse of the polynomial degree, for the p-version of the finite element method and spectral methods). Suppose that the spaces become more and more accurate, in the sense that

$$
\lim _{h \rightarrow 0} \inf _{v \in V_{h}}\|u-v\|=0 .
$$

Then if the stability constant $C_{h}$ remains bounded as $h \rightarrow 0$, it follows that $u_{h}$ converges to $u$ at the same rate as the best approximation. If, on the other hand, $C_{h} \rightarrow+\infty$ quickly enough, in general $u_{h}$ will not converge to $u$ at all as $h \rightarrow 0$. If $C_{h} \rightarrow+\infty$ slowly, then $u_{h}$ may still converge to $u$, but generally at a slower rate than the best approximation. In the case where $C_{h}$ stays bounded we say that our method is stable (here method refers to the whole sequence of $V_{h}$, i.e., includes the mesh refinement or degree enhancement procedure). In summary, if the method is stable, then the approximate solution converges to the exact solution at the same rate as the best approximation error.

Remark. This basic result can be extended in two ways to cover the majority of linear finite element applications. (Many further extensions are possible as well, including to nonlinear problems.) First, we have only considered Galerkin methods where the trial space (in which $u_{h}$ is sought) and the test space (over which the test function $v$ varies) are the same space $V_{h}$. The standard mixed methods are of this sort. However, the fundamental error bound above applies equally well to the case of Petrov-Galerkin methods where different spaces are used. Second, we have only considered conforming methods in which the discrete problem is to find $u_{h} \in V_{h}$ such that $B(u, v)=F(v)$ for all $v \in V_{h}$, with the space $V_{h} \subset V$. If $V_{h} \not \subset V$ or if we use an approximate bilinear form $B_{h}$ or an approximate linear form $F_{h}$ on the discrete level which is unequal to the corresponding exact form $B$ or $F$ (e.g., because of numerical quadrature), then the method is nonconforming. For nonconforming methods the discrete equations

$$
B_{h}\left(u_{h}, v\right)=F_{h}(v) \quad \text { for all } v \in V_{h}
$$

will in general not be satisfied by the exact solution $u$. The degree to which the exact solution fails to satisfy the discrete equations is called the consistency error. If the consistency error is appropriately quantified, the fundamental principle above extends to 
non-conforming methods as follows: if the method is stable, then the error in the Galerkin solution is bounded by a multiple of the sum of the approximation error and the consistency error. In this paper we will continue only to consider conforming methods, for which the consistency error is zero.

5. Stability of mixed methods. The basic theory sketched in the last section applies equally well to displacement methods and mixed methods. For example, consider again the elasticity problem, and for simplicity suppose that the boundary conditions are for vanishing displacement on the whole boundary $\left(\Gamma_{d}=\partial \Omega, g_{d}=0\right)$. For the primal formulation the bilinear form $B$ in (4) is then

$$
B(\mathbf{u}, \boldsymbol{v})=\int_{\Omega} C \mathcal{E}(\mathbf{u}): \mathcal{E}(\boldsymbol{v}) \quad \text { for } \mathbf{u}, \boldsymbol{v} \in \stackrel{\circ}{\mathbf{H}}^{1}
$$

( $\stackrel{\circ}{ }^{1}$ is the subspace of $\mathbf{H}^{1}$ of functions vanishing on the boundary), while for the mixed formulation of this problem

$$
B((\mathcal{S}, u),(\mathcal{T}, v))=\int_{O}(A \mathcal{S}: \mathcal{T}+\operatorname{div} \mathcal{T}: \mathbf{u}+\operatorname{div} \mathcal{S}: \boldsymbol{v}) \text { for }(\mathcal{S}, u),(\mathcal{T}, v) \in \mathcal{H}(\operatorname{div}) \times \mathrm{L}^{2}
$$

(cf. Table 2). A major difference between the two cases arises when we try to find finite element spaces which yield stable approximations. For displacement methods there is no difficulty. In fact any choice of subspaces $V_{h} \subset \stackrel{\circ}{\mathbf{H}}^{1}$ yields stable approximation. This is because the bilinear form (6) is coercive, that is, the inequality

$$
B(v, v) \geq \alpha\|v\|^{2} \quad \text { for all } v \in V
$$

holds for some positive constant $\alpha$. (This is ensured by Korn's inequality, which asserts the existence of such a constant $\alpha$ depending only on the domain $\Omega$. In fact the primal formulations for all our examples are coercive.) Now the discrete solution $u_{h} \in V_{h}$ is defined by the equations $B\left(u_{h}, v\right)=F(v)$ for $v \in V_{h}$. Setting $v=u_{h}$ and invoking coercivity and the definition of the dual norm $\|\cdot\|_{*}$, we get

$$
\alpha\left\|u_{h}\right\|^{2} \leq B\left(u_{h}, u_{h}\right)=F\left(u_{h}\right) \leq\|F\|_{*}\left\|u_{h}\right\|
$$

whence

$$
\left\|u_{h}\right\| \leq \alpha^{-1}\|F\|_{*}
$$

Thus the stability constant $C_{h}$ for this discretization is bounded by $1 / \alpha$ no matter how the subspace $V_{h}$ is chosen. Consequently the error will be of the same order as the error in best approximation. The choice of subspace need therefore only be guided by considerations of approximability and efficiency of implementation. In short, Galerkin methods based on coercive formulations are always stable.*

*Here we use the fact that the test and trial spaces are identical. Petrov-Galerkin methods based on coercive formulations are not necessarily stable. 
The situation for mixed methods is altogether different. For mixed methods the space $V$ decomposes as the product of two spaces $V=S \times W$ and $B$ has the special form

$$
B((s, u),(t, v))=a(s, t)+b(t, u)+b(s, v)
$$

with $a: S \times S \rightarrow \mathbb{R}$ and $b: S \times W \rightarrow \mathbb{R}$ bilinear. One consequence is that for mixed formulations the bilinear form is never coercive and stability is by no means automatic. In fact elements which are chosen without due regard to stability will usually prove to be unstable. For example, from a naive point of view the simplest, most appealing element for the Stokes problem is the linear velocity-constant pressure element shown in Figure 1a. However the stability constant for this element is $\infty$ and the resulting discrete system of equations is singular on most meshes. This element is completely useless.

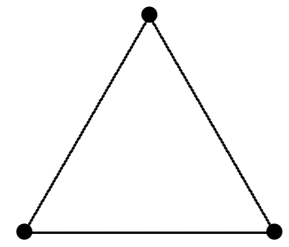

$\mathbf{u}$

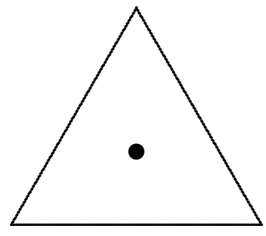

$p$

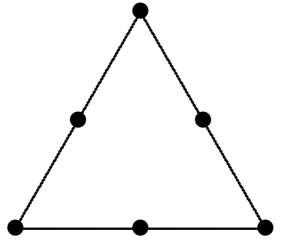

u

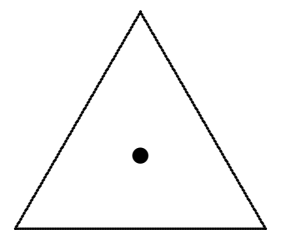

$p$

FIG. 1A. An unstable Stokes element.

FIG. 1B. A stable Stokes element.

For the Stokes problem the bilinear form $B$ takes the form (7) with

$$
\begin{gathered}
a(\mathbf{u}, \boldsymbol{v})=\int_{\Omega} \mathcal{C E}(\mathbf{u}): \mathcal{E}(\boldsymbol{v}) \quad \text { for } \mathbf{u}, \boldsymbol{v} \in \stackrel{\circ}{\mathbf{H}}^{1} \\
b(q, \boldsymbol{v})=\int_{\Omega} q \operatorname{div} \boldsymbol{v} \quad \text { for } q \in L^{2}, \boldsymbol{v} \in \stackrel{\circ}{\mathbf{H}}^{1}
\end{gathered}
$$

Note that in this case that, although $B$ is not coercive, at least $a$ is. In this case it can be shown that the stability constant may be bounded in terms of the reciprocal of the coercivity constant $\alpha$ for $a$ and the reciprocal of the quantity

$$
\beta_{h}=\inf _{v \in W_{h}} \sup _{s \in S_{h}} \frac{b(s, v)}{\|s\|\|v\|} .
$$

In particular if we choose a sequence of $S_{h}$ and $W_{h}$ for which $\beta_{h}$ stays bounded away from zero, the corresponding method is stable. The condition that $\beta_{h}$ stay bounded above zero is known as the second Brezzi condition or LBB condition.

Notice that as the space $S_{h}$ increases, for fixed $W_{h}$, the constant $\beta_{h}$ increases. In other words, for the Stokes problems (or, more generally, problems for which the $a$ form is coercive), enrichment of the space $S_{h}$ increases stability. For example the quadratic velocity-constant pressure Stokes element, shown in Figure 1b, is stable [14]. 
However the condition that the $a$ form be coercive is not satisfied for most mixed methods. In fact, of the four mixed formulations presented, only that for the Stokes problem has this property. For the elasticity problem, for example, we have $a(\mathcal{T}, \mathcal{T})=$ $\int_{\Omega} A \mathcal{T}: \mathcal{T}$. Since it is possible to find $\mathcal{T}$ which is bounded by 1 everywhere but for which the divergence of $\mathcal{T}$ is arbitrarily large, there cannot exist a constant $\alpha$ such that $a(\mathcal{T}, \mathcal{T}) \geq\|T\|_{\mathcal{H}(\mathbf{d i v})}^{2}$ for all $\mathcal{T}$ in $\mathcal{H}(\mathbf{d i v})$. So the $a$ form is indeed not coercive.

However, it turns out that one can get by with a weaker condition than coercivity of $a$ on $S$, namely coercivity on a particular subspace of $S_{h}$. More precisely, suppose that there exists a positive constant $\alpha_{h}$ such that

$$
a(z, z) \geq \alpha_{h}\|z\|^{2} \quad \text { for all } z \in Z_{h}
$$

where

$$
Z_{h}=\left\{z \in S_{h} \mid b(z, v)=0 \text { for all } v \in W_{h}\right\} .
$$

Then the stability constant may be bounded in terms of the reciprocals of the constants $\alpha_{h}$ in (9) and $\beta_{h}$ in (8). Thus if for a sequence of subspaces $S_{h} \times W_{h}$ the $\alpha_{h}$ remain bounded uniformly above zero (this is the first Brezzi condition), and the $\beta_{h}$ do likewise (second Brezzi condition), then the resulting method is stable. This is the content of Brezzi's theorem [10].

Let us briefly indicate the idea behind the theorem. Stability refers to the invertibility of the matrix representing the discrete problem, and the stability constant is the norm of the inverse matrix. For mixed methods, the matrix has the form

$$
\left(\begin{array}{cc}
\mathcal{A} & \mathcal{B}^{t} \\
\mathcal{B} & 0
\end{array}\right):\left\{\begin{array}{c}
S_{h} \\
W_{h}
\end{array}\right\} \rightarrow\left\{\begin{array}{c}
S_{h} \\
W_{h}
\end{array}\right\}
$$

The space $Z_{h}$ introduced above is the nullspace of the $\mathcal{B}$. Therefore, if we partition $S_{h}$ as $Z_{h} \times Z_{h}^{\perp}$, where $Z_{h}^{\perp}$ denotes the orthogonal complement of $Z_{h}$ in $S_{h}$, then the action of $\mathcal{B}$ on $S_{h}$ may be written as

$$
\left(\begin{array}{ll}
0 & \overline{\mathcal{B}}
\end{array}\right):\left\{\begin{array}{c}
Z_{h} \\
Z_{h}^{\perp}
\end{array}\right\} \rightarrow W_{h}
$$

where $\overline{\mathcal{B}}$ denotes the restriction of $\mathcal{B}$ to $Z_{h}^{\perp}$. Now the second Brezzi condition just asserts the invertibility of $\overline{\mathcal{B}}$. Similarly, let us decompose the action of $\mathcal{A}$ as, say,

$$
\left(\begin{array}{ll}
\overline{\mathcal{A}} & \mathcal{Q} \\
\mathcal{R} & \mathcal{S}
\end{array}\right):\left\{\begin{array}{l}
Z_{h} \\
Z_{h}^{\perp}
\end{array}\right\} \rightarrow\left\{\begin{array}{l}
Z_{h} \\
Z_{h}^{\perp}
\end{array}\right\}
$$

Thus $\overline{\mathcal{A}}$ is the matrix associated with the bilinear form $a$ restricted to $Z_{h} \times Z_{h}$, and the first Brezzi condition simply asserts the invertibility of this operator. The whole matrix (10), rewritten in terms of these new notations, is

$$
\left(\begin{array}{ccc}
\overline{\mathcal{A}} & \mathcal{Q} & 0 \\
\mathcal{R} & \mathcal{S} & \overline{\mathcal{B}}^{t} \\
0 & \overline{\mathcal{B}} & 0
\end{array}\right):\left\{\begin{array}{c}
Z_{h} \\
Z_{h}^{\perp} \\
W_{h}
\end{array}\right\} \rightarrow\left\{\begin{array}{c}
Z_{h} \\
Z_{h}^{\perp} \\
W_{h}
\end{array}\right\}
$$


or, rearranging rows and columns,

$$
\left(\begin{array}{ccc}
\overline{\mathcal{B}}^{t} & \mathcal{R} & \mathcal{S} \\
0 & \overline{\mathcal{A}} & \mathcal{Q} \\
0 & 0 & \overline{\mathcal{B}}
\end{array}\right):\left\{\begin{array}{c}
W_{h} \\
Z_{h} \\
Z_{h}^{\perp}
\end{array}\right\} \rightarrow\left\{\begin{array}{c}
Z_{h}^{\perp} \\
Z_{h} \\
W_{h}
\end{array}\right\}
$$

From the upper triangular form, it is clear that the invertibility of $\overline{\mathcal{B}}$ (which is equivalent to the invertibility of $\overline{\mathcal{B}}^{t}$ ) and the invertibility of $\overline{\mathcal{A}}$ are together are necessary and sufficient for the invertibility of the whole matrix.

While Brezzi's theorem furnishes us with relatively concrete conditions which yield stability, the verification of these conditions can be quite difficult. A number of analytic techniques have been developed that ease the task some what, for example, localization theorems [9], the use special mesh-dependent norms [7], etc. We shall not go into any of these techniques here, but in the next section we discuss a number of elements that have, in one way or another, been shown to be stable.

6. The construction of stable mixed elements. In $\S 4$ we saw that the accuracy of a finite element discretization is determined by the approximability of the exact solution by the finite element subspace and the stability of the discretization. These two properties, together with implementational issues, furnish the major factors for the construction and evaluation of the finite element spaces to be used. In $\S 5$ we saw that stability is automatic for coercive methods, such as most displacement methods, so that the finite element space can be chosen on the basis of approximation and ease of implementation alone. However, for mixed methods the question of stability is paramount.

Various techniques have been developed for the design of stable mixed elements. In this section we review some of these techniques and some of the resulting elements. We emphasize that this review is by no means exhaustive, neither with regard to the techniques nor to the resulting methods.

As remarked above, for the Stokes problem, in which the $a$ form is coercive, stability can always be achieved by adequate enrichment of the velocity space. There are a number of ways to enrich the space. For example, the unstable linear velocity-linear pressure Stokes element may be stabilized by the addition of a single internal velocity degree of freedom via a bubble. See Figure 2. This is the MINI element of Arnold, Brezzi, and Fortin [3]. A related element is the quadratic velocity-linear pressure Stokes element or Taylor-Hood element. By passing to quadratic velocities, not only is the element stable (on all but some very special mesh topologies), but a higher rate of convergence is achieved. The Taylor-Hood element was conceived independent of any proof of its stability, and verifying stability is much more difficult than for the MINI element or any of the other Stokes elements discussed in this section.

A second (closely related) method of enrichment is to use a finer mesh for velocity than pressure. For example, although the quadrilateral bilinear velocity-constant pressure 


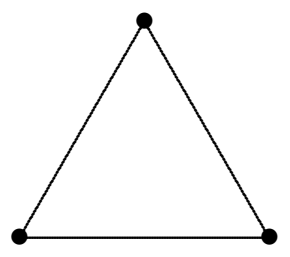

$\mathbf{u}$

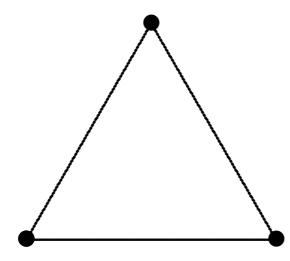

$p$

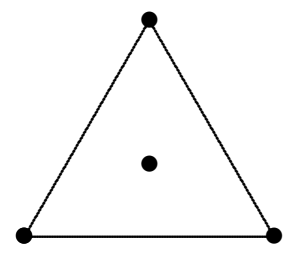

$\mathbf{u}$

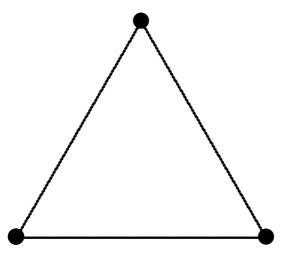

$p$

FIG. 2. An unstable Stokes element (left), stabilized by a bubble degree-of-freedom (right).

element is unstable (giving rise to the famous checkerboard pressure modes), it can be stabilized by using a composite velocity element which is bilinear on each of four quadrilateral microelements for each quadrilateral pressure element. See Figure 3.

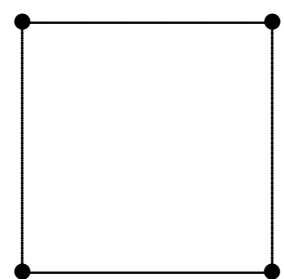

$\mathbf{u}$

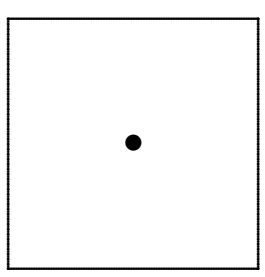

$p$

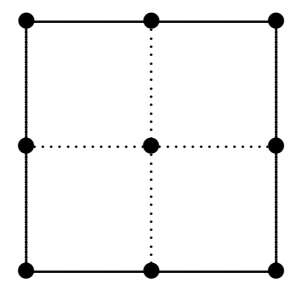

$\mathbf{u}$

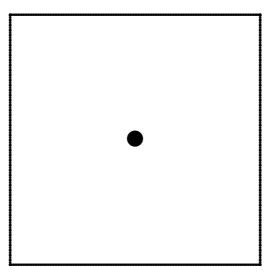

$p$

FIG. 3. An unstable Stokes element (left), stabilized by use of a composite velocity element (right).

Yet another method to stabilize a Stokes element is to enrich a conforming velocity space to a non-conforming one. This is the idea behind the Crouzeix-Raviart [11] method shown on the right hand side of Figure 4.

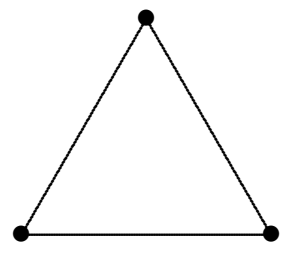

$\mathbf{u}$

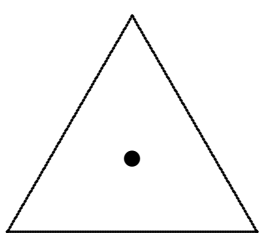

$p$

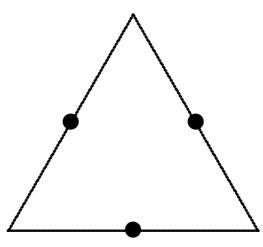

$\mathbf{u}$

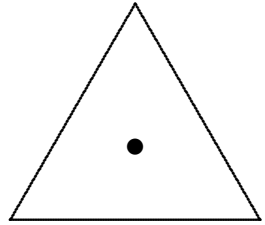

$p$

FIG. 4. Conforming linear velocity-constant pressure (left) is unstable, but non-conforming linear velocity-constant pressure (right) is stable.

Practically any Stokes element can be rendered stable by sufficient enrichment of the velocity space, the only limitation being the cost associated to the extra velocity degrees of freedom. If the original element afforded similar rates of approximation of velocity and pressure, the additional degrees of freedom for velocity won't increase the rate of convergence, since the approximation will be limited by the pressure. Thus, for instance, the quadratic velocity-constant pressure element pictured in Figure 1b rather disappointingly converges at the rate of the best approximation by linear velocity and constant pressure. 
For problems in which the $a$ form is not coercive (such as our other three examples), enrichment of the dual variable space need not improve stability. The difficulty is that as $S_{h}$ gets larger, so does $Z_{h}$, and hence the first condition (8) becomes harder to satisfy. Thus we are confronted with a trade-off that didn't appear for the Stokes problem. While bubbles, composite elements, and non-conforming elements have all been used in the development of elements for elasticity and other problems, their use is more subtle than for the Stokes problem. For example, Figure 5 shows two stable elements for plane elasticity, the first due to Johnson and Mercier [17], the second to Arnold, Douglas, and Gupta [4], each of which utilizes a composite element for the stress.

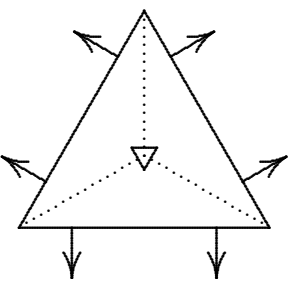

$\mathcal{S}$

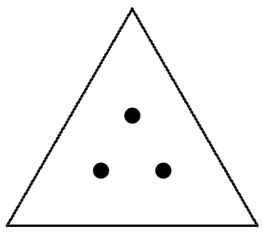

$\mathbf{u}$

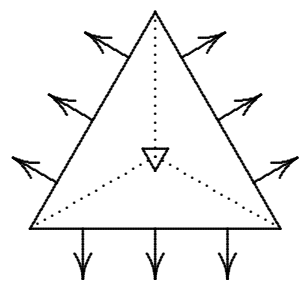

$\mathcal{S}$

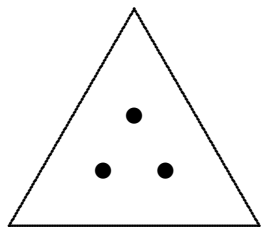

$\mathbf{u}$

Fig. 5. The Johnson-Mercier and Arnold-Douglas-Gupta elasticity elements, both of which are stable. The arrows represent the traction (two components). The triangles represent the stress tensor (three independent components). For the former element the stresses are piecewise linears with certain continuity across the dotted lines; for the latter piecewise quadratics are used.

Many mixed finite elements utilize the fact that the dual variable in three of our example problems is required to have square integrable divergence, but the entire gradient need not exist. That is, the dual variable is sought in $H$ (div) not in the smaller space $H^{1}$. For a piecewise polynomial function to belong to $H$ (div) it is not necessary that it be continuous across interelement boundaries (as it would have to be were it to lie in $H^{1}$ ). Only the normal component need be continuous - the tangential component is unconstrained. Thus finite element functions which are discontinous may nevertheless be conforming approximations of $H(\mathrm{div})$. This allows a certain flexibility which can be exploited in the construction of elements. Thus for example the Raviart-Thomas elements and the BrezziDouglas-Marini elements, the simplest cases of which are pictured in Figure 6, are of this sort. The latter element can be thought of as a means to stabilize the continuous linear flux-constant temperature element, which is unstable. It also uses linear elements for flux and constant elements for temperature, but the space for fluxes is increased by allowing functions with only the normal component continuous.

The flexibility afforded by using elements which are discontinuous in some components has also been applied to plate bending problems. The variational principle for the Kirchhoff-Love plate can be set up so that continuity is only required on the normal bending moment, $\mathbf{n}^{t} \mathcal{M} \mathbf{n}$, while the tangential and twisting moments can jump. The Hellan-Herrmann-Johnson element exploits this flexibility to enable the use of a piecewise constant approximation to the moment tensor. This element, which is diagrammed in Figure 7, has been shown to be stable in a specially devised, mesh-dependent norm [7]. 


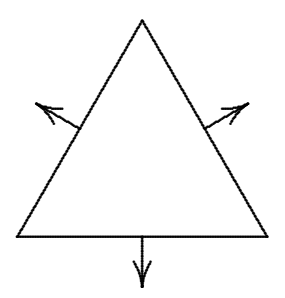

$\mathbf{S}$

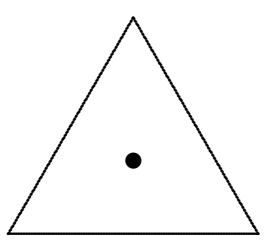

$u$

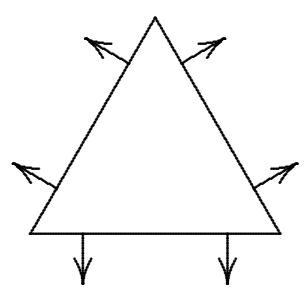

$\mathbf{S}$

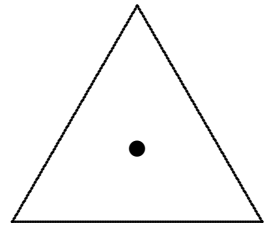

$u$

FIG. 6. The lowest order cases of the Raviart-Thomas and Brezzi-Douglas-Marini elements for scalar second order elliptic problems. The arrows represent the normal component of flux. The flux space for the Raviart-Thomas element consists of vectorfields of the form $(a+b x, c+b y)$ on each element. The flux space for the Brezzi-Douglas-Marini space is the full space of linear vectorfields on each element.

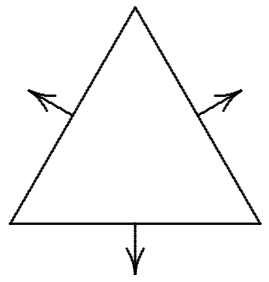

$\mathcal{M}$

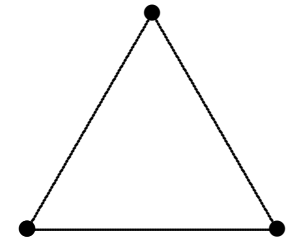

$w$

Fig. 7. The Hellan-Herman-Johnson plate bending element. The moment tensor is approximated by a piecewise constant function. The arrows represent the normal bending moment component on the edge. The transverse displacement is approximated by a continuous piecewise linear function.

Most of the examples presented above have been of elements of quite low order. In fact, it is usually easier to obtain stability with higher order elements. Thus, for example, the rather natural Stokes element based on continuous velocity elements of degree $d$ and discontinuous pressure elements of degree $d-1$ is unstable for $d=1,2$, or 3 , but can be shown to be stable for $d \geq 4$ [21]. (If discontinuous pressure elements are used, then stable elements are achieved for all degrees $d>1$, the case $d=2$ being the Taylor-Hood element.)

The stability constant depends jointly on the bilinear form $B$ (which, for mixed methods, is built of the two forms $a$ and $b$ ), and the trial space $V_{h}$ (which, for mixed methods, is built of the two spaces $S_{h}$ and $W_{h}$ ). Thus far we have discussed ways to construct and modify the trial space for a given bilinear form in order to obtain stability. It is possible to modify the bilinear form as well. For example, while the linear velocity-linear pressure Stokes element is not stable with the usual bilinear form, if the discrete solution is determined from the system

$$
\int_{\Omega}\left[C \mathcal{E}\left(\mathbf{u}_{h}\right): \mathcal{E}(\boldsymbol{v})+\operatorname{div} \boldsymbol{v} p_{h}+\operatorname{div} \mathbf{u}_{h} q-h^{2} \operatorname{grad} p_{h} \operatorname{grad} q\right]=\int_{\Omega} \mathbf{f} \cdot \boldsymbol{v}
$$

the resulting method is stable. This is the simplest example of a Galerkin-Least Squares method.* In this case the change in the bilinear form introduces a consistency error (which

*If the bubble degree of freedom is eliminated from the MINI element by static condensation, one arrives, essentially, at the same method. 
however is small enough not to affect the rate of convergence). It is also possible to modify the bilinear form in a consistent way and still have the linear/linear element stable [16]. In the last five years there have been numerous papers presenting extensions and variations of this procedure to obtain simple, stable mixed methods for a variety of problems.

An alteration of the mixed variational formulation for elasticity of an entirely different sort was introduced by Arnold and Falk [5]. They derived a variational principle involving the displacement field and a second-order tensorfield called the pseudostress, from which the true stress can easily be recovered as a linear combiniation of components. Their new variational principle is very similar to the Hellinger-Reissner principle but does not require a symmetry constraint on the tensorfield. This allows one to easily adapt mixed elements for the scalar second order elliptic problem, such as the Raviart-Thomas or Brezzi-Douglas-Marini elements described above.

\section{REFERENCES}

[1] D. N. ARnold, Discretization by finite elements of a model parameter dependent problem, Numer. Math., 37 (1981), pp. 405-421.

[2] D. N. ARnold And F. BREzzI, Mixed and nonconforming finite element methods: implementation, postprocessing and error estimates, Math. Modelling and Numer. Anal., 19 (1985), pp. 7-32.

[3] D. N. Arnold, F. Brezzi, and M. Fortin, A stable finite element for the Stokes equations, Calcolo, 21 (1984), pp. 337-344.

[4] D. N. Arnold, J. Douglas, And C. Gupta, A family of higher order mixed finite element methods for plane elasticity, Numer. Math., 45 (1984), pp. 1-22.

[5] D. N. ARnold ANd R. S. FAlK, A new mixed formulation for elasticity, Numer. Math., 53 (1988), pp. 13-30.

[6] I. BABUŠKa AND J. E. Osborn, Generalized finite element methods: their performance and their relation to mixed methods, SIAM J. Numer. Anal., 20 (1983), pp. 510-536.

[7] I. Babuška, J. E. Osborn, And J. Pitkäranta, Analysis of mixed methods using mesh-dependent norms, Math. Comp., 35 (1980), pp. 1039-1062.

[8] K. J. Bathe And F. BRezzi, On the convergence of a four-node plate bending element based on Mindlin/Reissner plate theory and a mixed interpolation, in Mathematics of Finite Elements and Applications V, J. R. Whiteman, ed., Academic Press, New York, NY, 1985, pp. 491-503.

[9] J. Boland and R. Nicolaides, Stability of finite elements under divergence constraints, SIAM J. Numer. Anal., 20 (1983), pp. 722-731.

[10] F. BREzzI, On the existence, uniqueness, and approximation of saddle point problems arising from Lagrangian multipliers, RAIRO Anal. Numér., 8-32 (1974), pp. 129-151.

[11] M. Crouzeix and P. -A. Raviart, Conforming and non conforming finite element methods for solving the stationary Stokes equations, RAIRO Anal. Numér., R3 (1973), pp. 33-76.

[12] B. Darlow, R. Ewing, and M. Wheeler, Mixed finite element methods for miscible displacement in porous media, Sixth SPE Symposium on Reservoir Simulation, SPE 10501, New Orleans, 1982.

[13] R. Ewing, T. Russell, And M. Wheeler, Simulation of miscible displacement using mixed methods and a modified method of characteristics, Seventh SPE Symposium on Reservoir Simulation, SPE 12241, San Francisco, 1983.

[14] M. Fortin, Calcul numérique des écoulements des fluides de Bingham et des fluides Newtoniens incompressible par la méthode es éléments finis, Univ. Paris, Thesis, 1972. 
[15] B. X. Fraeijs de Veubeke, Displacement and equilibrium models in the finite element method, in Stress Analysis, O. C. Zienkiewicz and G. Hollister, eds., John Wiley \& Sons, New York, NY, 1965.

[16] T. J. R. Hughes, L. P. Franca, and M. Balestra, A new finite element formulation for computational fluid mechanics: V. Circumventing the Babuška-Brezzi condition: a stable PetrovGalerkin formulation of the Stokes problem accounting for equal order interpolation, Comput. Methods Appl. Mech. Engrg., 59 (1986), pp. 85-99.

[17] C. Johnson And B. Mercier, Some equilbrium finite element methods for two-dimensional elasticity problems, Numer. Math., 30 (1978), pp. 103-116.

[18] C. Johnson and J. Pitkäranta, Analysis of some mixed finite element methods related to reduced integration, Math. Comp., 38 (1982), pp. 375-400.

[19] D. S. Malkus and T. J. R. Hughes, Mixed finite element methods-reduced and selective integration techniques: a unification of concepts, Comput. Methods Appl. Mech. Engrg., 15 (1978), pp. 63-81.

[20] L. D. Marini And A. SAVini, Accurate computation of electric field in reverse-biased semiconductor devices: a mixed finite element approach, Compel, 3 (1984), pp. 123-135.

[21] L. R. Scott And M. Vogelius, Norm estimates for a maximal right inverse of the divergence operator in spaces of piecewise polynomials, RAIRO Modél. Math. Anal. Numér., 19 (1985), pp. 111-143. 